\title{
Rationalization of urban road space by congestion: towards an alternative tool for travel demand management
}

\author{
C. A. González \& F. Robusté \\ School of Civil Engineering, Universidad Politécnica de Cataluña, Spain
}

\begin{abstract}
In this paper we discuss the subject matter of transport sustainability in urban environments as from an equitable use of the urban road space on behalf of the different transport modes. This paper provides the conceptual and methodological bases for the development of an alternative tool for transport demand management called "Spatial Rationalization Charge by Congestion (SRC)", which expresses the percentage of road space destined for private vehicles which has to be transferred to other transport modes of greater environmental efficiency (such as public transport and non motorized modes) according to the internalization of the externalities of the private vehicle.
\end{abstract}

Keywords: transport demand management, road pricing, congestion charge.

\section{Introduction}

The use of the private vehicle enters in conflict with the social welfare, because the externalities that the private vehicle generates exceed largely those of public transport; and more specifically, private transport requires more space on the road network and it generates more contamination per passenger than public transport. From the social point of view, this gives rise to the need of developing tools for the management of the road space, to give priority to public transport. But if public transport is favoured, and there is no penalty for the use of the private vehicle, the demand that public transport could attract from private transport would be replaced by a potential request for the private vehicle.

From the point of view of economic theory, the use of prices has been widely defined as the mechanism that guarantees an efficient use of infrastructures. After the pioneer task of Pigou [1], authors such as Walters [2], and Vickey [3], 
established the basis to fix a tax for the use of the infrastructure in accordance with the costs of congestion. Recently this idea has gained force in multiple theoretical and empirical reports, and today it is the policy established by the European Commission.

In spite of its technical coherence and its sufficient theoretical support, the introduction of the congestion tax always implies a high reticence of the citizens because it is a question of a monetary penalty and it also has a political cost for the local authorities that have to make the decisions. This is why there is the need to develop an alternative tool for traffic demand management as the one we present here, that no longer penalizes the private vehicle in monetary terms but in spatial terms, which not only has the same efficiency minimizing the generalized transport costs, but that is also coherent with the instructions for a more sustainable urban transport policy, and that has a greater social acceptance.

\section{Spatial rationalization charge by congestion -SRC-}

The SRC expresses the percentage of road space destined to the private vehicle that should be transferred to other modes of transport of greater environmental efficiency according to the internalisation of its social marginal costs. This penalty implies inducing an increment in the travel time to the private vehicle according to its social marginal costs (by means a reduction of its road space) that favours a more efficient traffic re-assignment on the network, and the change in the modal choice towards public transport. In other words, it is about the use of the congestion level as a regulator for modal distribution. As a first approach to the development of this alternative tool for traffic demand management, the "first best" is used as the pricing system, the considered externalities are limited to the traffic congestion, and a first analysis is presented for the simple case of two routes.

\subsection{Link between the diagrams, congestion tax and reduction of the offer of road space}

Considering that there exists a corresponding relation between the optimal tax, $\tau^{*}$, which is the result of the difference between the marginal social costs (social trip costs) and the average travel costs (private trip costs), and a variation of the travel time $\Delta t$, which results from the reduction of the road space, so that it is possible to establish a variation (increase) of the travel time $\Delta t$, according to an optimal tax $\tau^{*}$, which allows one to speak of a penalty for the user of a private vehicle, not only in monetary terms as happens with the traditional tools, but now in time units.

At a conceptual level, it is a matter of a displacement of the curve of offer $O$ towards an offer $O^{\prime}$, which states a reduction in the offer of road space destined for the private vehicle, the effect of which is an increase in the travel time in this mode of transport, and therefore a reduction in the demanded quantity (number of trips, or traffic flow) for this mode of transport. 
This displacement of the curve of offer generates a $\Delta t$ which is a variation (increase) of the travel time for the private vehicle. This induced delay is therefore the difference of the travel time for an initial offer (without external regulation) and the travel time for a future offer under optimization criterions, so that $\Delta t=t^{\prime}-t$.

Diagram $t / Q$. Reduction of the offer of road space for the private vehicle (Towards an alternative tool: the SRC).
Diagram $C / Q$. Average and marginal costs of travel for the private vehicle (Used for Congestion Pricing).
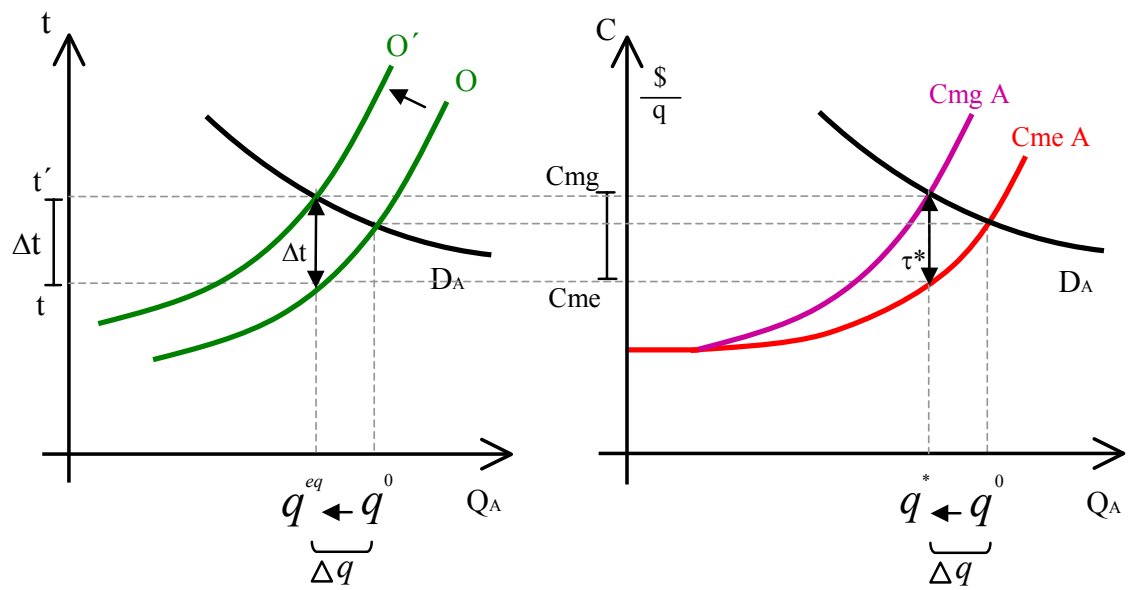

Figure 1: Tax by congestion and reduction of the offer of road space.

This $\Delta t$ represents the additional travel time with which the private vehicle should be penalized for the delays that an additional unit induces to the system. The aim is that the private vehicle should not only assume its average travel costs (or private travel costs), but also the marginal travel costs (or social travel costs), resulting in this way in an internalisation of the externalities. The $\Delta t$ we found will be the basis for the calculation of the spatial charge by congestion.

\subsection{The penalty for the private vehicle in time units: approach to the problem, mathematical formulation and resolution method}

We follow the methodological approach used in Daganzo [4], in which a simple model of supply and demand is used to explore the possibilities of an alternative strategy of control, to the classic pricing by congestion; as such, we consider a simple scenario of two routes that consists of only one congested link, and of other alternatives to go from an origin to a destination.

Four basic assumptions: one congested link (bottleneck); fixed time and cost for nonusers; linear utility; non-returned revenues. The assumptions allow us to express the extra utility of the bottleneck for a specific class $i$, as a function of 
the relative cost and time of the bottleneck $\left(c_{i} \mathrm{y} t_{i}\right)$ experienced by class members, the congestion status of the bottleneck $(T)$, and the toll paid $(\tau)$.

Specifically, the extra utility of the bottleneck for the user of specific class i, is:

$$
U_{i}=b_{c i}\left(\Delta c_{i}+\tau\right)+b_{t i}\left(\Delta t_{i}+T(q)\right)
$$

where $T(q)$ : time due to the congestion, when $\mathrm{q}$ trips choose to use the bottleneck; $\Delta c_{i}, \Delta t_{i}$ : differences in costs and time between the bottleneck and other modes when $\mathrm{q}=0$ and there is no tax; $b_{c i}, b_{t i}$ : these parameters are the marginal utilities of money and of time; $b_{t i} / b_{c i}$ : this ratio is the value of the time $\mathrm{i}$ for the class $\mathrm{i}$.

\subsubsection{One socioeconomic class: the latent demand}

It is convenient to describe a bivariable function, $q$, of time and money, which gives as a result the latent demand for the bottleneck as a function of the congestion time $T$, and the charged toll, $\tau$. By latent demand we are referring to the quantity of trips which would be in the disposition to use the bottleneck under specific conditions. Without a regulation, the latent demand is carried out, and becomes the flow $q$.

Since we assume that the utility is linear, which points out that the bottleneck is chosen by the class $\mathrm{i}$ if the relative utility in time units for the bottleneck is positive, then for those who use the bottleneck $C_{i}<0 \quad\left(U_{i}>0\right)$, the demand can be defined as the following probability function.

$$
\begin{aligned}
& q(\tau, T)=\operatorname{Pr}\left\{U_{i}>0\right\}=\operatorname{Pr}\left\{C_{i}<0\right\}= \\
& =\operatorname{Pr}\left\{-\left(\Delta c_{i} / \alpha+\Delta t_{i}\right)>T_{(q)}+\tau / \alpha\right\}
\end{aligned}
$$

The quantity $-\left(\Delta c_{i} / \alpha+\Delta t_{i}\right)$ from the previous equation, can be seen as a random variable $\mathrm{R}$, which represents the equivalent advantage in time units of the bottleneck when $q=0$ and $\tau=0$ (that is, as though the bottleneck was not congested). The quantity $T_{(q)}+\tau / \alpha$ can be interpreted as the penalty time due to the congestion and the tolls.

Thus, if we allow $F_{R}$ to denote the complementary function of distribution of the variable $\mathrm{R}$ (which is a decreasing function independent of the control strategy), then $q(\tau, T)$ can be expressed as:

$$
q(\tau, T)=F_{R}(T+\tau / \alpha)
$$

It establishes that, if the advantage in time (the saved time) of the bottleneck exceeds the penalty time implied by the congestion and the toll, $y=T+\tau / \alpha$, the users will choose to use the bottleneck; otherwise they will not do so. Since 
every user who chooses to use the bottleneck will be allowed to do so, the equation $q(\tau, T)=F_{R}(T+\tau / \alpha)$ gives us the flow $q$, of the bottleneck, the trips which are willing to use the bottleneck.

Like in Robusté and Sarmiento [5], here we will use an inverse function $G_{R}=F_{R}^{-1}$. Then, instead of expressing the demand as $q(\tau, T)$, it will be expressed as $p\left(t, T_{(q)}\right)$, that is, cost in time units according to $q$ (Figure 2).

$$
p\left(t, T_{(q)}\right)=G_{R}(T+\tau / \alpha)
$$

Figure 2 represents a hypothetic curve associating the resulting flow $q$ (or quantity of trips) with a penalty time $y$. This figure also shows a supply curve with a tax, expressed as $T$. A differential increase of the tax displaces the supply function $T$ in $\mathrm{d} \tau / \alpha$ time units. The elimination of the tax produces a displacement $-\tau / \alpha$, which gives a supply curve without a tax T- $\tau / \alpha$, and a point of equilibrium $\left(q_{0}, T_{1}\right)$. The intersection of the curves $G_{R}$ and $T$, and its projection on the curve without a tax is the short list $\left(q^{*}, T^{\prime \prime}, T^{*}\right)$.

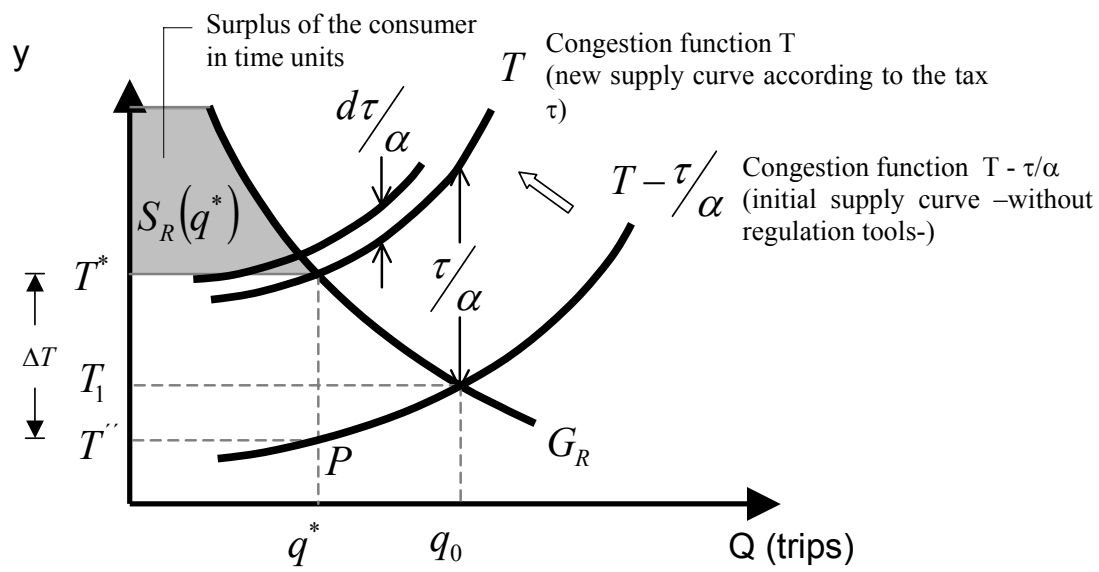

Figure 2: Equilibrium analysis with the new supply curve according to tax, and with costs in time units.

For the i class, the relative utility relative to the bottleneck expressed in time units is the difference between the time of advantage (the saved time) of the bottleneck, $r_{i}$, and the penalty time implied by the congestion and the toll, $y$. For the $i$ class, the utility of the bottleneck should not be negative -because negative differences between the time of advantage and the penalty time, result in a non use, this is:

$$
U_{i}=\max \left\{0, U_{i}\right\}=\max \left\{0, b_{c i} C_{i}\right\}=
$$




$$
\begin{gathered}
=\max \left\{0, b_{c i}\left[\left(\Delta c_{i}+\tau\right)+\alpha\left(\Delta t_{i}+T\right)\right]\right\} \\
U_{i}=\max \left\{0,-b_{t i}\left[-\left(\Delta c_{i} / \alpha+\Delta t_{i}\right)-(T+\tau / \alpha)\right]\right\}= \\
=\left(-b_{t i}\right) \max \left\{0,\left(r_{i}-y\right)\right\}
\end{gathered}
$$

where $r_{i}=-\left(\Delta c_{i} / \alpha+\Delta t_{i}\right)$ e $y=(T+\tau / \alpha)$

The relative utility of the bottleneck expressed in time units is:

$$
\max \left\{0, U_{i}\right\} /\left(-b_{t i}\right)=\max \left\{0,\left(r_{i}-y\right)\right\}
$$

Utility in time units for class $i=\max \left\{0,\left(r_{i}-y\right)\right\}$

The utility in time units for class $i$ in equilibrium is given by the previous equation: $U_{i}$ in time units $=\max \left\{0,\left(r_{i}-y\right)\right\}$, being $y=T^{*}$. It is clear that according to Figure 2, $T^{*}$ is increased by increasing the tax $\tau$; therefore, $U_{i}$ in time units $=\max \left\{0,\left(r_{i}-y\right)\right\}$ cannot grow for any $i$ when the tax $\tau$ is increased. The utility of all the users is reduced when a toll is introduced. (Notice that the prospect of $U_{i}$ in time units $=\max \left\{0,\left(r_{i}-y\right)\right\}$, through $i$, is the surplus of the consumer expressed in time units, given by the shady area above $T^{*}$ in Figure 2. This surplus of the consumer decreases when the $\operatorname{tax} \tau$ is increased.)

If we have different socioeconomic classes and $f_{i}$ is the fraction of users that belong to each class, the benefit -surplus for the consumer (user) in time units is $\sum\left(f_{i} \max \left\{0, U_{i}\right\}\right)$. Therefore, the benefit for the consumer (user) is: $-b_{t i} E[\max \{0,(R-y)\}]$.

The equilibrium is found when $y=T^{*}$, and the benefit for the consumer in time units, $S_{R}$, is:

$$
S_{R}=E\left[\max \left\{0,\left(R-T^{*}\right)\right\}\right]
$$

which is represented in Figure 2 by the shady area $S_{R}$ above the $y$-axis $T^{*}$, where $E$ is the operator of mathematical expectancy. This benefit for the consumer also can be written as $S_{R}=S_{R}\left(q^{*}\right)$, where $q^{*}$ is the $x$-axis of $T^{\prime \prime}+\tau / \alpha$. This benefit for the consumer diminishes when $\tau$ increases, as we can see in Figure 2, when the tax is increased $d \tau / \alpha$ equivalent time units.

The total social welfare is the area below the supply curve, and above the time $T^{*}$ between zero and $q^{*}$. This total social welfare in time units is:

$$
B=q^{*} \tau / \alpha+S_{R}\left(q^{*}\right)
$$


In Figure 2, we observe how a rise $\mathrm{d} \tau / \alpha$ of the tax produces a reduction $d T$ in $T^{\prime \prime}$ and a $d q$ in $q^{*}$. These reductions are related to the slope of the curve $T-\tau / \alpha$ at the point $\mathrm{P}$.

The change in the total social welfare is:

$$
B=(\tau / \alpha) d q-q^{*} d T
$$

This total social welfare is maximized at the point where there is no change (derivative zero):

$$
\begin{gathered}
\frac{d B}{d \tau}=0=\left[(\tau / \alpha) \frac{d q}{d \tau}\right]-\left(q^{*} \cdot \frac{d T}{d \tau}\right) \\
q^{*} \frac{d T}{d q}=\tau / \alpha \\
\alpha q^{*} \frac{d T}{d q}=\tau
\end{gathered}
$$

From the maximization process of the total social welfare, we can obtain $\tau / \alpha$, which is the equivalent to the penalty expressed now in time units, with a unitary value $\alpha$. This is: $\tau / \alpha=q d T / d q$.

\subsection{The reduction of road space for the private vehicle}

In the previous section we reached the expression $\tau / \alpha$ which is the penalty for the private vehicle in time units, which in figure 2 is denoted on the axis $y$ (hours) as $\Delta T=T^{*}-T^{\prime \prime}$. As from the established $\Delta T$, penalty in time units or additional delay which will have to be induced to the travel time of the private vehicle (see figure 2), now the correspondent reduction of the capacity $C$ destined to be used by the private vehicle is calculated. That is, to diminish the maximum admissible flow of the road $q_{m}$, reaching a future capacity $C^{1}$ as from an initial capacity $C^{0}$.

In accordance with figure 3 , given an initial capacity $C^{0}$, a flow $q_{1}$, has a travel time $t_{C^{0}}$, and when applying a reduction of the capacity $\Delta C$, until a future capacity $C^{1}$, then we have for the same flow $q_{1}$ a new travel time $t_{C^{1}}$, and consequently a variation (increase) of the travel time of the private vehicle $\Delta t$. (Notice that by applying $\Delta C$, the point $q_{0}$ where the area of congestion begins is displaced to the left.)

Figure 3 shows that the reduction of the capacity $\Delta C$ (or reduction of the road space destined to the private vehicle) on the horizontal axis will be the optimal when the resultant variation of the travel time $\Delta t$ on the vertical axis is equal to the previously calculated penalty in time units $\Delta T$ (in the y-axis of the figure 2). That is, the optimal point of reduction of road space destined to the private 
vehicle, with which the private vehicle internalises its marginal social costs by means of a penalty in time units added to its travel time, is achieved when $\Delta t=\Delta T$.

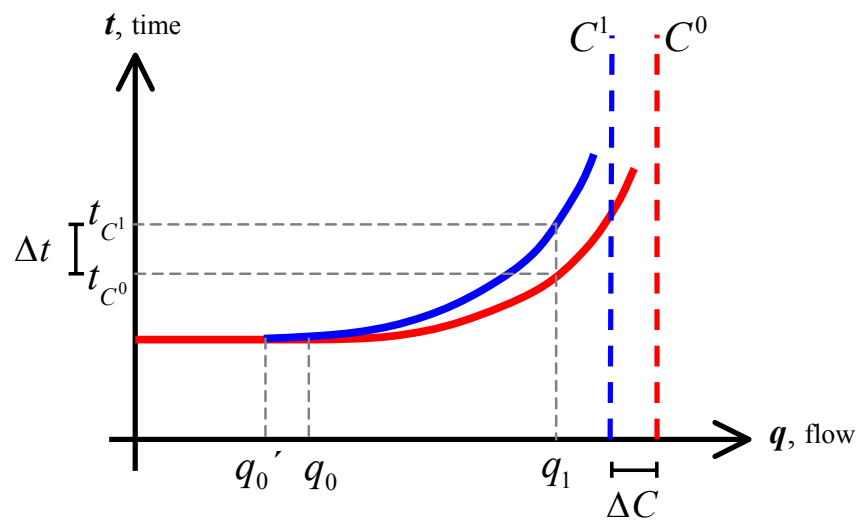

Figure 3: The physical phenomenon of congestion, and the reduction of the road capacity destined to the private vehicle.

In a practical way, what it would mean in a real setting is to identify, as from a given section of a road, the $n$ lanes destined to the private vehicle, which is here accepted as $C^{0}$, and later apply a reduction to this $n$ lanes by transferring it to another transport mode. Effectively we will work with a simple model of congestion: the link performance functions. While different formulations of such functions have been suggested over the years, the BPR function (BPR [6]) is very well suited for the use in conjunction with traffic assignment models. The BPR function relates link travel times as a function of the volume/capacity ratio according to:

$$
t=t_{f}\left[1+\alpha\left(\frac{v}{c}\right)^{\beta}\right]
$$

where $t$ : congested link travel time, $t_{f}:$ link free flow time, $v$ : link volume, $c$ : link capacity, $\alpha$ and $\beta$ : parameters.

\subsection{The implementation of the spatial rationalization charge by congestion}

To implement the SRC, we need to consider two main aspects: the minimum reduction and transference of road space unit (MRTU), and the assignment of this road space to other modes of transport of greater environmental efficiency. 
The first aspect, the one referred to the reduction and transference of road space units, implies to change from the use of continuous values (percentages) to discrete values (number of lanes), since the reduction and transference of road space is applied to the section of the road, and this has to finally be expressed in number of lanes which will be reduced from the total capacity of the road destined to the private vehicle, and later transferred to other transport modes.

Since each of the lanes destined to the private vehicle represents a percentage of the total number of lanes destined to the private vehicle, and this percentage per lane destined to the private vehicle is the basic unit of reduction and transference of road space (MRTU), the optimal $\Delta \mathrm{C}$ (that is, the reduction of the road space destined to the private vehicle until reaching an optimal point in function of the internalization of its externalities) will have to have at least a value close to 1 reduction and transference unit (MRTU), so that it justifies its implementation.

An optimal $\Delta \mathrm{C}$ with a value much lower than MRTU will indicate that the measure cannot be implemented, because by reducing the road capacity for the private vehicle by one lane we will fall into a high overcharge for the private vehicle, which is not justified in the microeconomic model of the spatial SRC, which considers -at least in principle- the congestion as the only externality to be taxed. However, even if in this paper we do not discuss it, it is possible to consider other additional externalities in the model, such as environmental pollution, noise, and number of accidents amongst others, which would diminish this overcharge for the private vehicle until reaching values which are more in agreement with the aim of internalizing the externalities.

The second aspect implies having a series of criterions for the assignment of this road space to other modes of transport. The aspects which have to be considered involve, in principle, the relation between the supply and demand of the road space for the other modes of transport on this road axis, and the activities (land uses) on this axis. Like this we can establish the suitability of transferring space to introduce a bus lane, a rail for tramway, a bicycle lane, or the enlargement of the pavement for pedestrians, or a mixture of the previous if it were needed (an other option is to consider the possibility of introducing a multiuse lane).

\section{Conclusions}

We consider that the alternative tool for traffic demand management which we present here can have similar results to the ones by road pricing with regard to the maximization of the social welfare, and it can have a greater acceptance on behalf of the citizens and the local administration: First, because it is not a direct monetary payment for the users, second because of its double function as a tool for sustainability in urban transport: optimal distribution of the use of the road network and promotion of a change in the modal choice towards public transport as an alternative of greater environmental efficiency; and third because its materialization is well used to carry out urban planning actions of improvement of the public space. 
The subject of transport sustainability in urban and metropolitan areas is approached here from the perspective of a more rational and efficient distribution of the use of the urban road space, which we consider the key to the discussion. The practical evidence shows us that an equitable distribution of the travellers according to the mode of transport is not sufficient, because even though private transport, the public transport and other not mechanized modes such as walking or cycling had equal parts of the total of trips carried out in a town, more than $80 \%$ of the network is being used by the private vehicle.

This indicates that it is on this last aspect where we have to concentrate our efforts, since on this depend the multiple externalities such as congestion, environmental pollution, noise, number of accidents amongst others, which have a direct connection with the volume of private vehicle traffic. It is in this framework that we present this alternative tool for traffic demand management as one tool more to be considered as part of the integral transport policies in urban and metropolitan areas.

\section{References}

[1] Pigou, A.C. The Economics of Welfare. First edition, McMillan, London, 1920.

[2] Walters, A.A. The theory and measurement of marginal private and social costs of highway congestion. Econometrica, 29, pp 676-699, 1961.

[3] Vickrey, W. Pricing in urban and suburban transport. American Economic Review: Paper and Proceedings, 53, pp 452-465, 1963.

[4] Daganzo, Carlos F. A pareto optimum congestion reduction scheme. Transportation Research B: Methodological. Vol 29, issue 2, pp 139-154, 1995.

[5] Robuste, F. \& Sarmiento, I. Road pricing systems in urban environments (In spanish). Ministerio de Fomento, 1999.

[6] BPR. Traffic assignment manual for application with a large, high speed computer. Washington: U. S. Dept. of Commerce Bureau of Public Roads Office of Planning, Urban Planning Division, 1964. 